\title{
Contention Control: A Game-Theoretic Approach
}

\author{
Lijun Chen, Steven H. Low and John C. Doyle \\ Engineering \& Applied Science Division, California Institute of Technology, USA
}

\begin{abstract}
We present a game-theoretic approach to contention control. We define a game-theoretic model, called random access game, to capture the contention/interaction among wireless nodes in wireless networks with contention-based medium access. We characterize Nash equilibria of random access games, study their dynamics and propose distributed algorithms (strategy evolutions) to achieve the Nash equilibria. This provides a general analytical framework that is capable of modelling a large class of systemwide quality of service models via the specification of per-node utility functions, in which systemwide fairness or service differentiation can be achieved in a distributed manner as long as each node executes a contention resolution algorithm that is designed to achieve the Nash equilibrium. We thus design medium access method according to distributed strategy update mechanism achieving the Nash equilibrium of random access game. In addition to guiding medium access control design, the random access game model also provides an analytical framework to understand equilibrium and dynamic properties of different medium access protocols and their interactions.
\end{abstract}

\section{INTRODUCTION}

Wireless channel is a shared medium that is interferencelimited. Contention-based medium access control (contention control) is a distributed strategy to access and share wireless channel among contending wireless nodes. From a controltheoretic point of view, it consists of two components: a contention resolution algorithm that dynamically adjusts persistence probability or contention window in response to contention in the network, ${ }^{1}$ and a feedback mechanism that updates a contention measure and sends it back to wireless nodes. Different medium access control methods differ in terms of how they adjust persistence probability or contention window in response to contention and what contention measure they use. For example, the standard IEEE 802.11 DCF uses a backoff mechanism and a binary contention signal - packet collision or successful transmission, in which each wireless node doubles its contention window upon a collision (binary exponential backoff) and sets it to the base value upon a successful transmission [10].

The choice of contention measure and contention resolution algorithm is key to the performance of medium access methods. "Inappropriate" choice of these two components will result in poor performance. For example, in high-load scenarios, 802.11 DCF results in excessive collisions and

This work is partially supported by NSF through grants CNS-0435520 and CCR-0326554, DARPA through grant HR0011-06-1-0007, and Boeing.

${ }^{1}$ Contention resolution is usually achieved through two mechanisms: persistence and backoff [18]. In the persistence mechanism, each wireless node maintains a persistence probability and accesses the channel with this probability when it perceives an idle channel. In the backoff mechanism, each wireless node maintains a contention window and waits for a random amount of time bounded by the contention window before a transmission. hence low throughput, because setting to the base contention window upon successful transmissions is too drastic and each new transmission starts with the base contention window independent of the contention level in the network. It also has short-term unfairness problem, due to the oscillation in contention window. The binary exponential backoff directly causes short-term unfairness. However, this oscillation in contention window is unavoidable because DCF uses a binary contention signal. In order to achieve high efficiency (high throughput and low collision) and better fairness, we need to stabilize the network into a steady state which sustains an appropriate contention window size (or equivalently, persistence probability) for each node.

The main motivation of this work is to provide an analytical model to study the contention/interation among wireless nodes and design medium access methods that could stabilize the network around a steady state with a target fairness (or service differentiation) and high efficiency. To this end, we define a general game-theoretic model, called random access game, to capture the contention/interaction among wireless nodes in wireless networks with contentionbased medium access. Here the game-theoretic model is not intended to model selfish behaviors of the wireless nodes, but rather to capture the constraints encountered in real networks. In real networks, we prefer distributed algorithms with no or minimal explicit message passing, and each wireless node does not know how many nodes are present, is not aware of the actions (such as transmission or channel access probability) of others a priori, and can only sense limited information about the channel state (such as packets encountering collisions, or channel being idle or busy). In such a situation, the best a node can do is to optimize some local or private objective and adjust its action based on limited information about the network state. Noncooperative game is best to model such a situation, and we design random access game to guide individual nodes to seek an equilibrium that achieves some systemwide performance objective.

In random access games, a player (wireless node) strategy is its persistence probability or equivalently its contention window size, and its payoff function includes both utility gain from channel access and cost from packet collision. Through the specification of per-node utility function, we can model a large class of systemwide quality of service models, similar to that in utility framework for network flows, see, e.g., [14]. We characterize the Nash equilibrium of random access games, study their dynamics and propose algorithms (strategy evolutions) to achieve the Nash equilibrium. We show that systemwide fairness or service differentiation can be achieved in a distributed manner as long as each node 
executes a contention resolution algorithm that is designed to achieve the Nash equilibrium.

Based on the understanding of the equilibrium and dynamics of random access games, we propose a novel medium access method derived from CSMA/CA in which each node estimates its conditional collision probability and adjusts its persistence probability or equivalently contention window accordingly. Unlike other medium access methods, our method adapts to continuous feedback signal (conditional collision probability) rather than binary contention signal (packet collision or successful transmission), and each node tries to keep a fixed persistence probability or equivalently contention window specified by the Nash equilibrium of random access game. By specifying appropriate utility functions, the resulting medium access method can achieve target fairness (or service differentiation), and better contention control (collision reduction) and hence higher throughput. In addition to guiding medium access control design, the random access game model also provides an analytical framework to understand equilibrium and dynamic properties of different medium access protocols and their interactions.

\section{RANDOM ACCESS GAME}

Consider a set $N$ of wireless nodes in a wireless LAN with contention-based medium access. We will focus on singlecell wireless LANs where every node can hear every other node in the network, see [6] for the extension to multicell wireless LANs. We consider the case of greedy nodes, i.e., they always have a frame to transmit. We will mainly present our theory in terms of "channel access probability." If a persistence mechanism is implemented, the channel access probability is just the persistence probability. If a backoff mechanism is implemented, channel access probability $p$ is related to a constant contention window $\mathrm{cw}$ according to $p=\frac{2}{c w+1}$. This relation can be derived under the decoupling approximation for a set of wireless nodes with constant contention windows, see, e.g., [3] [15].

Assume that each node $i \in N$ attains a utility $U_{i}\left(p_{i}\right)$ when it accesses the channel with probability $p_{i} \in\left[\nu_{i}, \omega_{i}\right]$. We assume that $U_{i}(\cdot)$ is continuously differentiable, strictly concave, increasing, and with the curvatures bounded away from zero in $\left[\nu_{i}, \omega_{i}\right]$, i.e., $-1 / U_{i}^{\prime \prime}\left(p_{i}\right) \geq 1 / \lambda>0$. Let $q_{i}(\mathbf{p}):=1-\prod_{j \neq i}\left(1-p_{j}\right)$ denote the conditional collision probability of node $i$. Our objective is to choose $\mathbf{p}:=\left(p_{1}, p_{2}, \ldots, p_{|N|}\right)$ such that each node maximizes its payoff $U_{i}\left(p_{i}\right)-p_{i} q_{i}$. Since wireless nodes are not aware of channel access probabilities of others a priori, we model their interaction as a noncooperative game. Formally, we define a random access game as follows.

Definition 1: A random access game $\mathcal{G}$ is defined as a triple $\mathcal{G}:=\left\{N,\left(S_{i}\right)_{i \in N},\left(u_{i}\right)_{i \in N}\right\}$, where $N$ is a set of players (wireless nodes), player $i \in N$ strategy $S_{i}:=$ $\left\{p_{i} \mid p_{i} \in\left[\nu_{i}, \omega_{i}\right]\right\}$ with $0 \leq \nu_{i}<\omega_{i} \leq 1$, and payoff function $u_{i}(\mathbf{p}):=U_{i}\left(p_{i}\right)-p_{i} q_{i}(\mathbf{p})$ with $q_{i}(\mathbf{p}):=1-\prod_{j \neq i}\left(1-p_{j}\right)$.

Note that the throughput of node $i$ is proportional to $p_{i}$ if there is no collision, and $p_{i} q_{i}$ is the collision probability experienced by node $i$ and can be seen as the "cost" resulting from collision. Thus, the payoff function $u_{i}(\cdot)$ has a nice interpretation: the net gain of utility from channel access, discounted by the cost due to collision.

Random access game $\mathcal{G}$ is defined in a rather general manner. Each node $i$ can choose any utility function $U_{i}(\cdot)$ it thinks appropriate. If all nodes have the same utility functions, the system is said to have homogeneous users. If the nodes have different utility functions, the system is said to have heterogeneous users. The motivation for studying systems of heterogeneous users is to provide differentiated services to different wireless nodes.

\section{A. Nash Equilibrium}

We now analyze the equilibrium of random access game. The solution concept we use is the Nash equilibrium [8]. Denote the strategy (channel access probability) selection for all nodes but $i$ by $\mathbf{p}_{-i}:=$ $\left(p_{1}, p_{2}, \ldots, p_{i-1}, p_{i+1}, \ldots, p_{|N|}\right)$, and write $\left(p_{i}, \mathbf{p}_{-i}\right)$ for the strategy profile $\left(p_{1}, p_{2}, \ldots, p_{i-1}, p_{i}, p_{i+1}, \ldots, p_{|N|}\right)$. A vector of access probability $\mathbf{p}^{*}$ is a Nash equilibrium if, for all nodes $i \in N, u_{i}\left(p_{i}^{*}, \mathbf{p}_{-i}^{*}\right) \geq u_{i}\left(p_{i}, \mathbf{p}_{-i}^{*}\right)$ for all $p_{i} \in S_{i}$. We see that the Nash equilibrium is a set of strategies for which no player has an incentive to change unilaterally. The following result is immediate.

Theorem 2: There exists a Nash equilibrium for any random access game $\mathcal{G} .^{2}$

Since utility function $U_{i}(\cdot)$ is concave, at the Nash equilibrium, $p_{i}^{\star}$ either takes value at the boundaries of the strategy space $S_{i}$ or satisfies

$$
U_{i}^{\prime}\left(p_{i}^{*}\right)=q_{i}\left(\mathbf{p}^{*}\right)
$$

where $U_{i}^{\prime}\left(p_{i}^{*}\right)=\frac{d U_{i}\left(p_{i}^{*}\right)}{d p_{i}}$, the marginal utility at $p_{i}^{*}$. We call a Nash equilibrium $\mathbf{p}^{*}$ a nontrivial equilibrium if, for all nodes $i, p_{i}^{*}$ satisfies equation (1), and trivial equilibrium otherwise. In the remainder of this section, we will mainly focus on nontrivial Nash equilibria.

Theorem 3: Random access game $\mathcal{G}$ has a nontrivial Nash equilibrium if the following condition holds:

A1: For each node $i \in N$, inverse function $\left(U_{i}^{\prime}\right)^{-1}\left(q_{i}\right)$ maps any $q_{i} \in[0,1]$ into a point $p_{i} \in S_{i}$.

The assumption A1 gives a sufficient condition for the existence of nontrivial Nash equilibrium. Since $U_{i}\left(p_{i}\right)$ is a continuously differentiable concave function, $U_{i}^{\prime}\left(p_{i}\right)$ is a continuous, decreasing function and so is $\left(U_{i}^{\prime}\right)^{-1}\left(q_{i}\right)$. Without loss of generality, with the assumption A1 we constrain the strategy space $S_{i}$ such that $\left(U_{i}^{\prime}\right)^{-1}(0)=\omega_{i}$ and $\left(U_{i}^{\prime}\right)^{-1}(1)=\nu_{i}$ in the following discussion.

Define idle probability $\gamma(\mathbf{p}):=\prod_{i \in N}\left(1-p_{i}\right)$, and $\Gamma_{i}\left(p_{i}\right):=\left(1-p_{i}\right)\left(1-U_{i}^{\prime}\left(p_{i}\right)\right)$. It follows from equation (1) that, at nontrivial Nash equilibrium,

$$
\Gamma_{i}\left(p_{i}^{*}\right)=\gamma\left(\mathbf{p}^{*}\right)
$$

Note that the right-hand side of the above equation is independent of $i$. Thus, $\Gamma_{i}\left(p_{i}^{*}\right)=\Gamma_{j}\left(p_{j}^{*}\right)$ for any $i, j \in N$.

\footnotetext{
${ }^{2}$ See [6] for complete proofs for all theorems in this paper.
} 
Theorem 4: Suppose A1 holds. Random access game $\mathcal{G}$ has a unique nontrivial Nash equilibrium if additionally the following condition holds:

A2: $\Gamma_{i}\left(p_{i}\right)$ is a monotone function in $S_{i}$ for all $i \in N$.

In order to study quality of service differentiation among wireless nodes, we further differentiate among symmetric and asymmetric equilibria as follows.

Definition 5: A Nash equilibrium $\mathbf{p}^{*}$ is said to be a symmetric equilibrium if $p_{i}^{*}=p_{j}^{*}$ for all $i, j \in N$, and an asymmetric equilibrium otherwise.

For a general system of homogeneous users, both symmetric and asymmetric Nash equilibria are possible. By symmetry, if a system of homogeneous users has an asymmetric Nash equilibrium, all its permutations are Nash equilibria. However, for symmetric nontrivial equilibrium, it must be unique.

Theorem 6: For a system of homogeneous users, if random access game $\mathcal{G}$ has symmetric nontrivial Nash equilibrium, it must be unique. More generally, for a system with several classes of homogeneous users, if $\mathcal{G}$ has symmetric nontrivial Nash equilibrium, ${ }^{3}$ it must be unique.

Since by symmetry there must be multiple asymmetric Nash equilibria if there exists any, the following result follows directly from Theorems 4 and 6 .

Corollary 7: For a system of homogeneous users, suppose $\mathrm{A} 1$ and $\mathrm{A} 2$ hold, then random access game $\mathcal{G}$ has a unique nontrivial Nash equilibrium which is a symmetric equilibrium. More generally, for a system with several classes of homogeneous users, under the same assumptions, $\mathcal{G}$ has a unique nontrivial Nash equilibrium which is symmetric among each class of users.

Corollary 7 is a powerful result. It guarantees the uniqueness of nontrivial Nash equilibrium, and moreover, it guarantees fair sharing of wireless channel among the same class of wireless nodes and provides service differentiation among different classes of wireless nodes. This will facilitate the analysis of dynamic property of random access games and the design of medium access control.

\section{B. Dynamics of Random Access Game}

The dynamics of game studies how interacting players could converge to a Nash equilibrium. It is a difficult problem in general, as pointed out in [8] that "game theory lacks a general and convincing argument that a Nash outcome will occur." In the setting of random access, players (wireless nodes) can observe the outcome (packet collision or successful transmission) of the actions of others, but do not have direct knowledge of other player actions and payoffs. We consider repeated play of random access game, and look for update mechanism in which players repeatedly adjust strategies in response to observations of other player actions so as to achieve the Nash equilibrium.

We consider a strategy update mechanism called gradient play [7]. In gradient play, every player adjusts a current

\footnotetext{
${ }^{3}$ For a system with several classes of users, a Nash equilibrium is symmetric if at equilibrium the users of the same class choose the same strategy.
}

channel access probability gradually in a gradient direction suggested by observations of other player actions. Mathematically, each node $i \in N$ updates its strategy according to

$$
p_{i}(t+1)=\left[p_{i}(t)+f_{i}\left(p_{i}(t)\right)\left(U_{i}^{\prime}\left(p_{i}(t)\right)-q_{i}(\mathbf{p}(t))\right)\right]^{S_{i}},
$$

where the stepsize $f_{i}(\cdot)>0$ can be a function of the strategy of player $i$, and " $S_{i}$ " denotes the projection onto the player $i$ strategy space. The gradient play admits a nice economic interpretation, by considering the conditional collision probability $q_{i}$ as contention price for node $i$. If the marginal utility $U_{i}^{\prime}\left(p_{i}\right)$ is greater than contention price, we increase the access probability, and if the marginal utility is less than contention price, we decrease the access probability. The following result is immediate.

Lemma 8: By the definition of nontrivial Nash equilibrium, nontrivial Nash equilibria of random access game $\mathcal{G}$ are fixed points of the gradient play (3) and vice versa.

Theorem 9: Suppose A1 and A2 hold, the gradient play (3) converges to the unique nontrivial Nash equilibrium of random access game $\mathcal{G}$ if for any $i \in N$, the stepsize $f_{i}\left(p_{i}\right)<$ $\frac{1}{\lambda+|N|-1}$.

Proof: By Lyapunov method using Lyapunov function $V(\mathbf{p}):=\sum_{i \in N}\left(U_{i}\left(p_{i}\right)-p_{i}\right)-\prod_{i \in N}\left(1-p_{i}\right)$. See [6] for details.

Theorem 9 guarantees the convergence of distributed gradient play to the desired Nash equilibrium. If a backoff mechanism is implemented, each node $i \in N$ updates its contention window $c w_{i}$ as follows:

$$
c w_{i}(t)=\frac{2-p_{i}(t)}{p_{i}(t)} .
$$

Remark: For a general game model, there may exist multiple nontrivial Nash equilibria. In this situation, a "naive" strategy such as best response or gradient play may not converge to the desired equilibrium, and concepts from control theory may come in to work, see, e.g., [19].

\section{Medium Access Control Design}

Corollary 7 and Theorem 9 suggest that random access games provide a general analytical framework to model a large class of systemwide quality of service models (mainly in terms of throughput) via the specification of per-node utility functions, and systemwide fairness or service differentiation can be achieved in a distributed manner as long as each node executes a contention resolution algorithm that is designed to achieve the Nash equilibrium.

Based on this understanding of the equilibrium and dynamics of random access games, we propose a novel medium access method derived from CSMA/CA: instead of executing exponential backoff upon collisions, each node estimates its conditional collision probability and adjusts its channel access probability and contention window according to gradient play (3) and (4), see Table I for a formal description. Unlike other medium access methods, our method adapts to continuous feedback signal (conditional collision probability) rather than binary feedback (packet collision), and stabilizes 
the network around a steady state specified by the Nash equilibrium of random access game. Our access method is an equation-based control, and its performance (such as throughput, collision and fairness) is determined by the Nash equilibrium. Note that $U_{i}^{\prime}\left(p_{i}(t)\right)-q_{i}(\mathbf{p}(t))$ specifies how far the current state is from the equilibrium. The contention window adjustment is small when the current state is close to the equilibrium and large otherwise, independent of where the equilibrium is. This is in sharp contrast to the approach taken by $802.11 \mathrm{DCF}$, where window adjustment depends on just the current window size and is independent of where the current state is with respect to the target equilibrium. So, our access method can achieve better contention control (collision reduction) and better short-term fairness. By specifying appropriate utility functions, the resulting access method can achieve better balance/tradeoff between channel access and collision avoidance, and hence a higher throughput.

TABLE I

MEDIUM ACCESS METHOD VIA GRADIENT PLAY

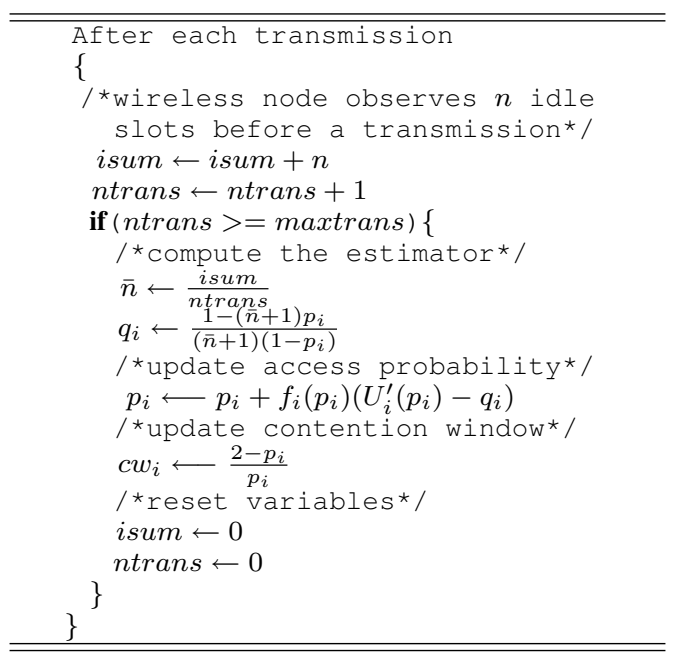

Furthermore, wireless nodes can estimate conditional collision probabilities by observing idle period of the channel. Let $n$ denote the number of consecutive idle slots between two transmissions. Here "a transmission" corresponds to a busy period in the channel when only a node transmits (i.e., a successful transmission) or multiple nodes transmit simultaneously (i.e., a collision). Since $n$ has the geometric distribution with parameter $\gamma(\mathbf{p})$, its mean $\bar{n}$ is given by $\bar{n}=\frac{\gamma(\mathbf{p})}{1-\gamma(\mathbf{p})}$. Thus, each node can estimate its conditional collision probability by observing the average number of consecutive idle slots, according to

$$
q_{i}=1-\frac{\gamma(\mathbf{p})}{1-p_{i}}=\frac{1-(\bar{n}+1) p_{i}}{(\bar{n}+1)\left(1-p_{i}\right)} .
$$

So, our access method can decouple contention control from handling packet losses, and is immune to those problems incurred in methods that infer channel contention from packet collisions.

In the next section, we will study a concrete random access game and the corresponding medium access control design, as a case study for the proposed design methodology in game-theoretic framework.

\section{A CASE STUDY}

Consider the following utility

$$
U_{i}\left(p_{i}\right):=\frac{1}{a_{i}}\left(\frac{\left(a_{i}-1\right) \omega_{i}}{a_{i}} \ln \left(a_{i} p_{i}-\omega_{i}\right)-p_{i}\right),
$$

where $0<\omega_{i}<1, a_{i}>1$ and $p_{i} \in\left[2 \omega_{i} /\left(1+a_{i}\right), \omega_{i}\right]$. Define a random access game $\mathcal{G}_{1}$ in the same way as in Definition 1 with the above player utility functions and strategy spaces.

\section{A. Equilibrium and Dynamics}

We have the following result regarding the Nash equilibrium of random access game $\mathcal{G}_{1}$.

Theorem 10: If $a_{i} \omega_{i}<1$, random access game $\mathcal{G}_{1}$ has unique nontrivial Nash equilibrium. Moreover, for a system of homogeneous users the unique nontrivial Nash equilibrium of $\mathcal{G}_{1}$ is a symmetric equilibrium, and for a system with several classes of homogeneous users the unique nontrivial Nash equilibrium of $\mathcal{G}_{1}$ is symmetric among each class of users.

Assume that each node $i \in N$ adjusts its strategy according to gradient play

$$
\begin{aligned}
p_{i}(t+1) & =\left[p_{i}(t)+f_{i}\left(p_{i}(t)\right)\left(\frac{\omega_{i}-p_{i}(t)}{a_{i} p_{i}(t)-\omega_{i}}-q_{i}(\mathbf{p}(t))\right)\right]^{S_{i}}, \\
c w_{i}(t) & =\frac{2-p_{i}(t)}{p_{i}(t)}
\end{aligned}
$$

The following result follows directly from Theorem 9.

Theorem 11: Suppose $a_{i} \omega_{i}<1$, the system described by equation (7) converges to the unique nontrivial Nash equilibrium of random access game $\mathcal{G}_{1}$ if $f_{i}\left(p_{i}\right)<\frac{1}{\lambda+|N|-1}$.

The condition $a_{i} \omega_{i}<1$ is a mild assumption and admits a very large region in parameter space. The Nash equilibrium can be easily calculated numerically. Note that $\Gamma_{i}\left(p_{i}\right)=$ $\frac{\left(1-p_{i}\right)\left(\left(1+a_{i}\right) p_{i}-2 \omega_{i}\right)}{a_{i} p_{i}-\omega_{i}}$ is a decreasing function of $\omega_{i}$ and an increasing function of $a_{i}$. Since $\Gamma_{i}$ is an increasing function of $p_{i}$, larger value of $\omega_{i}$ and/or smaller value of $a_{i}$ will results in larger channel access probability $p_{i}^{*}$ at equilibrium. Thus, in order to provide differentiated services, we can choose larger value of $\omega_{i}$ and/or smaller value of $a_{i}$ for the users of a higher priority class. For example, in wireless access network, we can assign a large $\omega_{i}$ value and/or small $a_{i}$ value to the access point, because usually downlink traffic is greater than the traffic of mobile nodes.

\section{B. Medium Access Control Design}

We design a medium access method according to channel access probability and contention window update algorithm (7)-(8), by modifying a Carrier Sense Multiple Access/Collision Avoidance (CSMA/CA) access method such as 802.11 DCF (see reference [10] for for a description of the channel access mechanism in DCF). As described in subsection II-C, our medium access method makes two key 
modifications to 802.11 DCF. Instead of adjusting contention window $c w_{i}$ to a binary feedback signal and using exponential backoff algorithm, each node $i$ estimates its conditional collision probability $q_{i}$, which is a continuous feedback, and adjusts $c w_{i}$ according to algorithm (7)-(8).

There are several parameters in our medium access method. The parameters $\omega_{i}$ and $a_{i}$ determine the equilibrium properties such as throughput, loss (collision) and fairness. The parameters $f_{i}(\cdot)$ and maxtrans determine the dynamical properties such as stability and responsiveness. In practice, we will choose a constant stepsize for all nodes (for example, a constant stepsize that accommodates a large range from several to tens of nodes in a single cell). The number of transmissions, maxtrans, for each node before updating its channel access probability and contention window, affects the convergence speed and the accuracy of the conditional collision probability estimation. Since by gradient play nodes update $p_{i}$ and $c w_{i}$ gradually, in order to achieve a good tradeoff between convergence speed and estimation accuracy we will choose a relatively small value for maxtrans and estimate average number of consecutive idle slots between transmissions using an exponential weighted running average

$$
\bar{n} \longleftarrow \beta \bar{n}+(1-\beta) \frac{\text { isum }}{\text { ntrans }},
$$

where $\beta \in[0,1)$. If $\beta$ is small we weight history less, and if $\beta$ is large we weight history more. By choosing appropriate $\beta$ value, exponential weighted running average gives better estimate than the "naive" estimator isum/ntrans.

\section{Performance}

To evaluate the performance of our medium access method, we develop a discrete-event simulator that implements our method and the standard 802.11 DCF basic access method. The values for the parameters used to obtain numerical results are as follows. The system values are those specified in the 802.11b standard with DSSS PHY layer [10]. In all simulations, we use a data rate of $11 \mathrm{Mbps}$ and packet payload of 12000 bits, and set the following values of the control parameters: maxtrans $=10, f_{i}=0.01$ and $\beta=0.2$. Also, the physical layer is assumed to be perfect for the numerical experiments reported in this subsection.

1) Throughput and Collision Overhead: We consider a system of homogeneous users, and compare the throughput achieved by our method and DCF. In our design each node $i$ is limited to choose a contention window size between $\frac{2-\omega}{\omega}$ and $\frac{1+a-\omega}{\omega}$, corresponding to channel access probability $p_{i} \in$ $\left[\frac{2 \omega}{a+1}, \omega\right]$. To compare the performance of our design with that of DCF on the same ground, we choose values for those related parameters such that $\frac{2-\omega}{\omega}=C W \min$ and $\frac{1+a-\omega}{\omega}=$ $2^{m} C W \max$, corresponding to a maximum backoff stage $m$. In our numerical experiments with DCF we also assume that after a packet's $(m+1)$ th failed transmission the contention window resets to $C W \min$. This is also equivalent to the packet being discarded after $m$ failed retransmissions.

Figure 1 and Figure 2 show the comparison of aggregate throughput and collision overhead between our design $(\omega=$
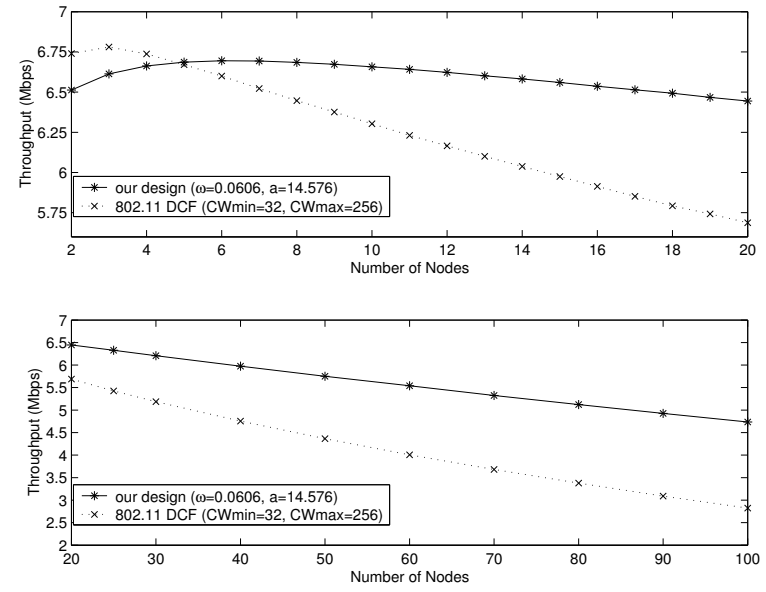

Fig. 1. Throughput comparison.
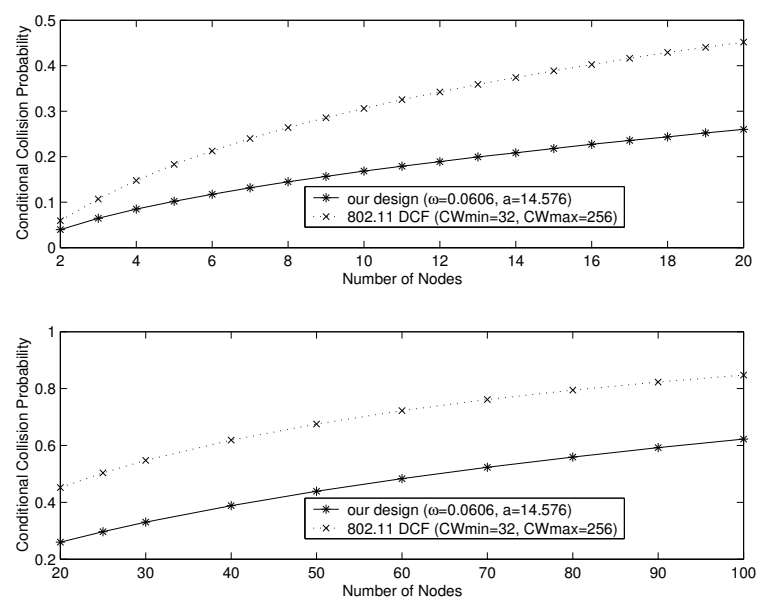

Fig. 2. Conditional collision probability comparison.

0.0606, $a=14.576)$ and DCF $(C W \min =32, C W \max =$ 256). We see that our access method achieves better tradeoff between channel access and collision avoidance, and hence higher throughput that is sustainable over a large range of numbers of competing nodes. We then compare the numerical values from simulations with the analytical values of throughput and conditional collision probability that are calculated with Nash equilibrium of random access game $\mathcal{G}_{1}$, and confirm that they match extremely well. This proves that our medium access method does converge to the desired Nash equilibrium of the random access game. We also track the evolution of contention windows, which approach quickly to and stay around the values specified by the Nash equilibrium.

2) Fairness: In our access method for a system of homogeneous users, wireless nodes have the same contention window size, specified by the symmetric Nash equilibrium of random access game $\mathcal{G}_{1}$. Thus, it is expected to have a better short-term fairness. Figure 3 compares short-term fairness of our access method and DCF using Jain fairness index for the window sizes that are multiples of the number of wireless nodes [12]. We can see that our method provide better short- 
term fairness than 802.11 DCF.

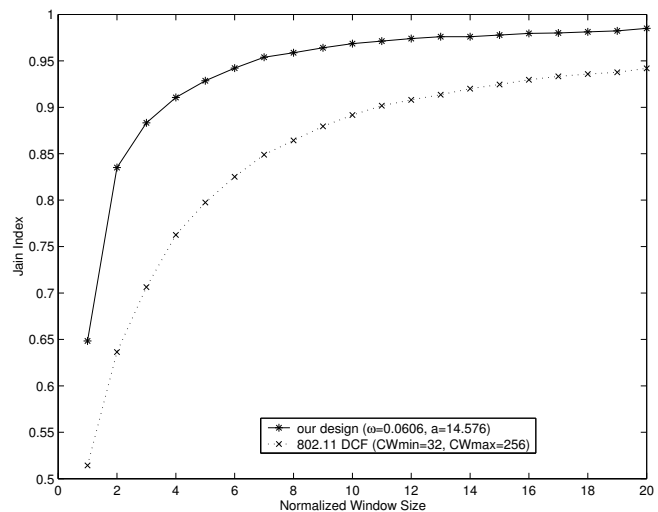

Fig. 3. Fairness comparison for 40 competing nodes.

3) Service Differentiation: As discussed before, we can provide service differentiation by choosing different utility functions for different classes of users. Regarding the concrete medium access method we consider, each node $i$ will receive different services by choosing different values for parameters $\omega_{i}$ or $a_{i}$. For the simplicity of presentation, we consider two classes of users. Assume that class 1 has $n_{1}$ users with parameters $\left(\omega_{1}, a_{1}\right)$, corresponding to a higher priority of service, and class 2 has $n_{2}$ users with parameters $\left(\omega_{2}, a_{2}\right)$, corresponding to a lower priority of service. Let first study the impact of $\omega_{i}$ on the service differentiation by setting the same $a_{i}$ value. The upper panel in Figure 4 shows the throughput ratio of a class 1 node to a class 2 node versus the total number of nodes for two different scenarios: two classes have equal number of users, and class 1 has fixed number of users. We see that, as the total number of nodes increase, the throughput ratio approaches 1.5. Indeed, a simple calculation can show that the throughput ratio between users of different classes is approximately $\frac{\omega_{1}}{\omega_{2}}$ for a large number of users. We then study the impact of $a_{i}$ on the service differentiation by setting the same $\omega_{i}$ value. The lower panel in Figure 4 shows the throughput ratio of a class 1 node to a class 2 node versus the total number of nodes for the scenario where two classes have equal number of users and the scenario where class 1 has fixed number of users. We see that, as the total number of nodes increase, the throughput ratio seems to converge to some fixed value. Again, a simple calculation can show that the throughput ratio will approach $\frac{1+a_{2}}{1+a_{1}}$.

\section{UTILITY FUnCTION AND REVERSE ENGINEERING}

As we see from the above discussions, utility functions determine Nash equilibria of random access games and thus the equilibrium (steady) operating points of medium access control protocols. Conversely, utility functions are determined by the equilibrium (steady) operating points of medium access control protocols. Since the medium access control protocol adapts channel access probability $p_{i}$ according to current access probability and packet collision, the equilibrium operating point defines an implicit relation
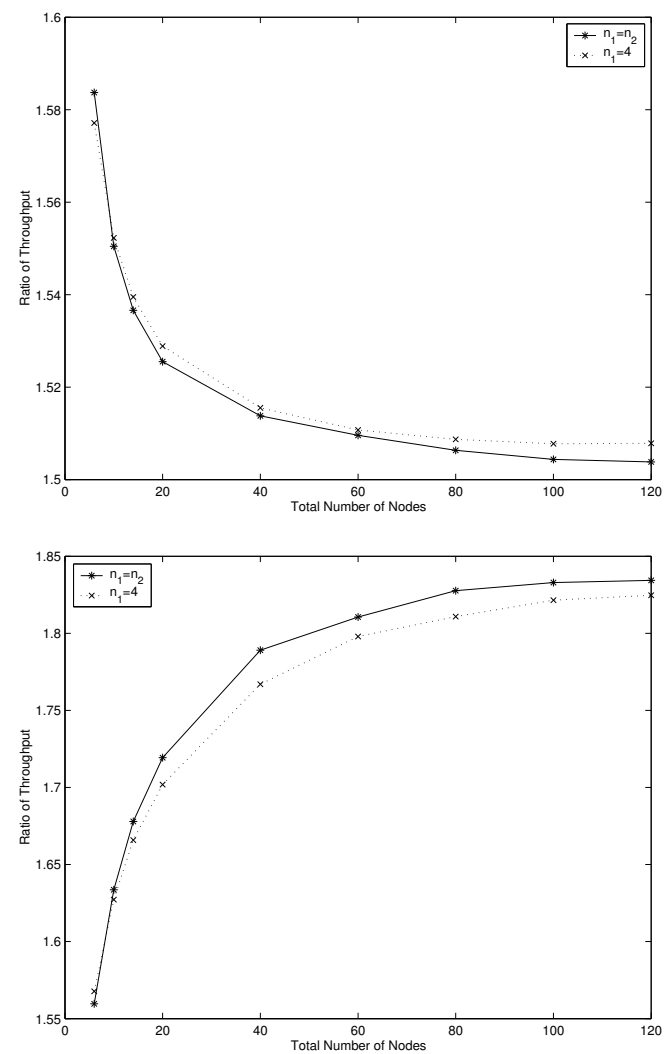

Fig. 4. The throughput ratio of a class 1 node to a class 2 node. Upper panel: $\left(\omega_{1}=0.06, a_{1}=15\right)$ and $\left(\omega_{2}=0.04, a_{2}=15\right)$; Lower panel: $\left(\omega_{1}=0.04, a_{1}=10\right)$ and $\left(\omega_{2}=0.04, a_{2}=20\right)$.

between equilibrium channel access probability $p_{i}$ and conditional collision probability $q_{i}$,

$$
p_{i}=\mathcal{F}_{i}\left(p_{i}, q_{i}\right) .
$$

Assume $\mathcal{F}_{i}$ is continuously differentiable and $\partial \mathcal{F}_{i} / \partial q_{i} \neq 0$ in $[0,1]$. Then, by implicit function theorem, there exists a unique continuously differentiable function $F_{i}$ such that

$$
q_{i}=F_{i}\left(p_{i}\right) .
$$

Define the utility function of each node $i$ as

$$
U_{i}\left(p_{i}\right)=\int F_{i}\left(p_{i}\right) d p_{i}
$$

With the above defined utility functions, we can define a random access game as in section II. Hence, we can reverse engineer medium access control protocols and study them in game theory framework: medium access control can be interpreted as a distributed strategy update algorithm to achieve the Nash equilibrium of the random access game.

For example, if we are first given the medium access method presented in subsection III-B, it can be interpreted as a distributed strategy update algorithm to achieve the Nash equilibrium of random access game $\mathcal{G}_{1}$ that is defined with the utility functions determined by the equilibrium of equation (7). Take another example, 802.11 DCF. It is well established that for a single-cell wireless LAN at steady state, 
channel access probability $p$ relates to conditional collision probability as follows [3]:

$$
p=\frac{2(1-2 q)}{(1-2 q)(C W \min +1)+q C W \min \left(1-(2 q)^{m}\right)},
$$

where $C W \min$ is the base contention window and $m$ is the maximum backoff stage. Following procedures (10)-(11) to derive a utility function, we can define a random access game and interpret DCF as distributed strategy update algorithm to achieve the corresponding Nash equilibrium. Note that, however, the dynamics of 802.11 DCF cannot be described by gradient play.

The random access game model can be used to analyze equilibrium properties such as throughput, collision and fairness of different medium access control protocols. When wireless nodes in a wireless LAN deploy different medium access protocols with different contention measures, we can also study the coexistence and interaction of different protocols in the random access game framework.

\section{RELATED WORK}

Much effort has been devoted to applying game-theoretic approach to study random access, see, e.g., [13] [17] [4] [5] [20]. The work closest to ours is Jin et al. [13] that studies noncooperative equilibrium of Aloha networks and their local convergence, and Borkar et al. [4] that studies distributed scheme for adapting random access. Our motivation, model and results are different from those work. Čagalj et al. [5] studies selfish behavior in CSMA/CA networks and propose a distributed protocol to guide multiple selfish nodes to a Pareto-optimal Nash equilibrium. In contrast, we use gametheoretic model to capture the information and implementation constraints encountered in real networks and design games to guide distributed users to achieve systemwide performance objectives. Another major difference of our work from most other game-theoretic works is that we take a control-theoretic viewpoint and regard channel access probabilities as dynamic variables. As such, we define a general utility for each user directly in terms of its channel access probability, and specify a special structure for random access game that respects the distributed and adaptive nature of contention-based medium access.

There are many papers on various improvements to 802.11 DCF that propose better contention resolution algorithms, see, e.g., [2] [16]. Our design is different in terms of both contention measure and contention resolution algorithm. There also exists extensive work on 802.11 QoS provisioning, see, e.g., [1] [11]. Our access method can provide more flexible service differentiations through the specification of per-node utility functions, except for manipulating the length of inter-frame space. Related work also includes [9] that proposes an idle sense access method, which compares the mean number of idle slots between transmission attempts with the optimal value and adopts an additive increase and multiplicative decrease algorithm to dynamically control the contention window in order to improve throughput and short-term fairness. In our access method, the number of consecutive idle slots between transmissions is only used to estimate conditional collision probabilities.

\section{Conclusions}

We have presented random access game model to study the contention/interaction among wireless nodes, and proposed to design medium access method according to distributed strategy update mechanism achieving the Nash equilibrium of random access game. As a case study of medium access control design in game-theoretic framework, we present a concrete medium access method and show that it achieves superior performance over the standard 802.11 DCF, and can provide flexible service differentiations among wireless nodes. In addition to guiding medium access control design, the random access game model also provides an analytical framework to understand equilibrium and dynamic properties of different medium access protocols and their interactions.

\section{REFERENCES}

[1] I. Aad and C. Castelluccia, Differentiation mechanisms for IEEE 802.11, Proceedings of IEEE Infocom, April 2001.

[2] I. Aad, Q. Ni, C. Barakat and T. Turletti, Enhancing IEEE 802.11 performance with slow $\mathrm{CW}$ decrease, IEEE 802.11e working group document 802.11-02/674r0, November 2002.

[3] G. Bianchi, Performance analysis of the IEEE 802.11 distributed coordination function, IEEE Journal on Selected Areas in Communications, 18(3):535-547, March 2000

[4] V. S. Borkar and A. A. Kherani, Random access in wireless ad hoc networks as a distributed game, Proceedings of WiOpt, March 2004.

[5] M. Čagalj, S. Ganeriwal, I. Aad and J. P. Hubaux, On selfish behavior in CSMA/CA networks, Proceedings of IEEE Infocom, March 2005.

[6] L. Chen, S. Low and J. Doyle, Random access game and medium access control design, Caltech Technical Report, 2007. http://www.cds.caltech.edu/ chen/papers/ramac.pdf

[7] S. D. Flam, Equilibrium, evolutionary stability and gradient dynamics, International Game Theory Review, 4(4):357-370, December 2002.

[8] D. Fudenburg and J. Tirole, Game Theory, The MIT Press, 1991.

[9] M. Heusse, F. Rousseau, R. Guillier and A. Dula, Idle sense: An optimal access method for high throughput and fairness in rate diverse wireless LANS, Proceedings of ACM Sigcomm, August 2005.

[10] IEEE, Wireless LAN media access control (MAC) and physical layer (PHY) specifications, IEEE Standard 802.11, June 1999.

[11] IEEE 802.11 task group e. http://grouper.ieee.org/groups/802/11/Reports/tge_update.htm.

[12] R. Jain, D. Chiu and W. Hawe, A quantitative measure of fairness and discrimination for resource allocation in shared computer systems, DEC Research Report TR-301, September 1984.

[13] Y. Jin and G. Kesidis, Equilibria of a noncooperative game for heterogeneous users of an Aloha networks, IEEE Communication Letters, 6(7):282-284, July 2002.

[14] F. P. Kelly, A. K. Maulloo and D. K. H. Tan, Rate control for communication networks: Shadow prices, proportional fairness and stability, Journal of Operations Research Society, 49(3):237-252, 1998.

[15] A. Kumar, E. Altman, D. Miorandi and M. Goyal, New insights from a fixed point analysis of single cell IEEE 802.11 WLANs, Proceedings of IEEE Infocom, March 2005.

[16] Y. Kwon, Y. Fang and H. Latchman, A novel MAC protocol with fast collision resolution for wireless LANs, Proceedings of IEEE Infocom, April 2003.

[17] A. B. MacKenzie and S. B. Wicker, Stability of multipacket slotted Aloha with selfish users and perfect information, Proceedings of IEEE Infocom, April 2003.

[18] T. Nandagopal, T. E. Kim, X. Gao and V. Bharghhavan, Achieving MAC layer fairness in wireless packet networks, Proceedings of ACM MobiCom, August 2000.

[19] J. S. Shamma and G. Arslan, Dynamic fictitious play, dynamic gradient play, and distributed convergence to Nash equilibria, IEEE Transactions on Automatic Control, 50(3):312-327, March 2005.

[20] A. Tang, J. W. Lee, J. Huang, M. Chiang and A. R. Calderbank, Reverse engineer MAC, Proceedings of WiOpt, April 2006. 